Gynäkologe 2020 · 53:560-561

https://doi.org/10.1007/s00129-020-04651-x

(C) Springer Medizin Verlag GmbH, ein Teil von Springer Nature 2020

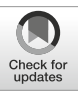

\author{
Anton Scharl ${ }^{1,3} \cdot$ Nicole Sänger ${ }^{2}$ \\ ${ }^{1}$ Klinik für Frauenheilkunde und Geburtshilfe, Klinikum St. Marien, Amberg, Deutschland \\ ${ }^{2}$ Gynäkologische Endokrinologie und Reproduktionsmedizin, Universitätsklinikum Bonn, Bonn, \\ Deutschland \\ ${ }^{3}$ Klinik für Frauenheilkunde und Geburtshilfe, Kliniken Nordoberpfalz AG - Klinikum Weiden, Weiden in \\ der Oberpfalz, Deutschland
}

\title{
Reproduktionsmedizin, eine gesellschaftliche Aufgabe
}

widmen wir diesem Thema dann einen Kongressband von Der Gynäkologe?

Neben Gynäkologie und Onkologie, Geburtshilfe und Perinatalmedizin ist die Gynäkologische Endokrinologie und Reproduktionsmedizin die dritte Säule und Thema eines Weiterbildungsschwerpunkts unseres Fachs. Und dennoch führt sie gefühlt eher eine Existenz am Rande, zumindest aus Sicht eines Klinikers. Sie ist $\mathrm{zu}$ einem ambulanten Fach geworden. Das ist nicht per se etwas Schlechtes. Aber es schafft eine schwierige Situation für Forschung und Weiterbildung, wenn das Gebiet nicht mehr an jeder Universitätsklinik vertreten ist. Die Gesetzeslage in Deutschland tut ein Übriges. Die Nationale Akademie der Wissenschaften Leopoldina mahnt dringend eine zeitgemäße Gesetzgebung für die Fortpflanzungsmedizin an. Nach ihrer Einschätzung zwingt die heute geltende, 30 Jahre alte Gesetzeslage (Embryonenschutzgesetz [ESchG] von 1990) die Ärzte nicht selten zu einer dem heutigen internationalen medizinischen Stand nicht mehr angemessenen Behandlung und führt zu unnötigen Risiken für Mutter und Kind. Mit anderen Worten, Deutschland wird auf diesem Gebiet abgehängt.

\section{1) Das seit 1990 bestehende ESchG muss dringend aktualisert werden}

Es wird also Zeit, dass wir die Reproduktionsmedizin erneut als ein dringendes Handlungsfeld für Frauenärztinnen und
Frauenärzte begreifen, nicht nur für die praktische Anwendung, sondern auch hinsichtlich der gesellschaftlichen Diskussion und der politischen Gestaltung.

Die immense Ausweitung des technisch Machbaren erfordert eine breite, ergebnisoffene, medizinisch-wissenschaftliche und ethisch-moralische Diskussion unter Beteiligung von uns Frauenärztinnen und Frauenärzten. Dabei geht es aber nicht nur um das Erlaubte, es geht auch darum, was menschlich und ethisch geboten ist, und es braucht klare rechtliche Regelungen.

In seiner 984. Sitzung hat der Bundesrat am 20. Dezember 2019 den „Entwurf eines Gesetzes zur Verbesserung der Hilfen für Familien bei Adoption“ beraten. Die Möglichkeit der Embryonenspende ist darin nicht erwähnt. Das ist bedauerlich und wenig verständlich, schließlich hat der Deutsche Ethikrat bereits 2016 empfohlen, die Embryonenspende rechtlich zu regeln. „Überzählige" Embryonen sind ein Nebenprodukt künstlicher Befruchtungen; eine Spende an Kinderwunschpaare ist aufgrund einer „Gesetzeslücke“ nicht verboten, auch ethisch nicht bedenklich, aber eine gesetzliche Regelung überfällig. Den langen Weg durch die Institutionen, der bei der Umsetzung von ethisch Gebotenem und rational Sinnvollem zu beschreiten ist, beschreiben Hans-Peter Eiden und Angelika Eder im Namen des Netzwerks Embryonenspende in ihrem Beitrag.

Während die Embryonenspende zwar nicht geregelt, aber doch nicht verboten ist, ist die Situation bei der Eizellspende klar: Sie ist verboten. Auch hier hat 
die Leopoldina dringenden Reformbedarf angemahnt. Heribert Kentenich und Klaus Diedrich beschreiben sehr differenziert die derzeitige Situation in Deutschland.

In der Technik der Reproduktionsmedizin gilt es, die Verfahren zu verbessern, die Risiken und Belastungen für die Frauen zu vermindern und die Erfolgschancen zu verbessern. Die Reduktion der endokrinen Belastung im Rahmen der Stimulation der Eizellreifung ist dazu ein Weg. Michael von Wolff und Isotta Magaton beschreiben diese Entwicklung. Sie zeigen gleichzeitig auf, welchen immensen Einfluss die Gesetzgebung und die Kostenerstattung auf die Chancen für praktische Umsetzung haben. Auch hier zeigt sich: Forschung und medizinisches Arbeiten können nur bedingt erfolgreich sein, wenn wir die (sozial)rechtlichen Rahmenbedingungen nicht ebenso konsequent bearbeiten.

\section{Korrespondenzadresse}

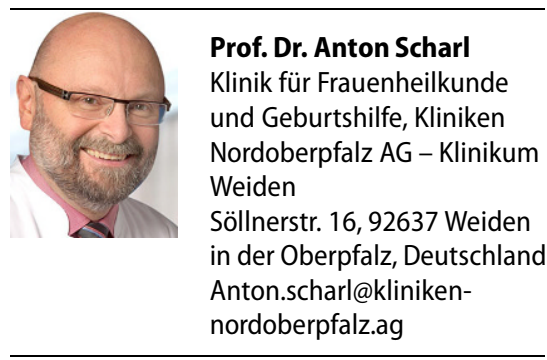

Interessenkonflikt. A. Scharl und N. Sänger geben an, dass kein Interessenkonflikt besteht.

\section{Aktuelle Buchempfehlungen aus dem Springer-Verlag}

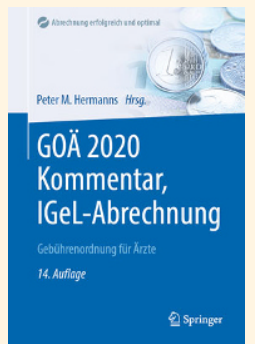

GOÄ 2020 Kommentar, IGeL-Abrechnung

Gebührenordnung für Ärzte

Hermanns, Peter M. (Hrsg.)

XXIV, 896 Seiten

2020| 14. Auflage

Springer-Verlag

978-3-662-60547-9 (ISBN)

$79,99 €$

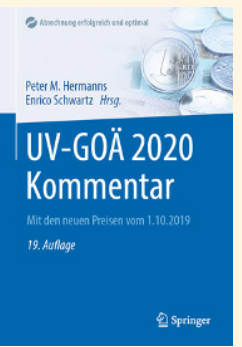

\section{UV-GOÄ 2020 Kommentar}

Mit den neuen Preisen vom 1.10.2019

Hermanns, Peter M., Schwartz, Enrico (Hrsg.)

XXIII, 767 Seiten

$2020 \mid$ 19. Auflage

Springer-Verlag

978-3-662-60549-3 (ISBN)

$69,99 €$

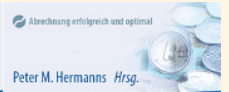

\section{EBM 2020 Kommentar Kinderheilkunde}

Kompakt: Mit Punktangaben, Eurobeträgen, Ausschlüssen, GOÄ

EBM 2020

Kommentar

Kinderheilkunde

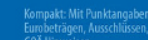

GOR Hinmesen
Unter Mitribet ion

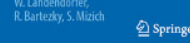

Springe

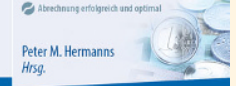

EBM 2020

Kommentar

Allgemeinmedizin

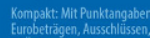

Gou Hirumeisen
Unter Mitarbeit פ⿴囗大 Springer

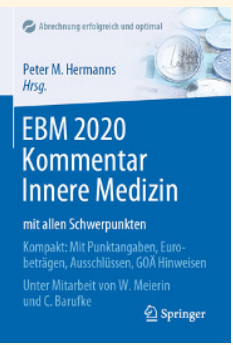

EBM 2020 Kommentar Allgemeinmedizin

Kompakt: Mit Punktangaben, Eurobeträgen, Ausschlüssen, GOÄ Hinweisen

Hermanns, Peter M. (Hrsg.)

$X X, 411$ Seiten

2020| 1. Aufl. 2020

Springer-Verlag

978-3-662-61501-0 (ISBN)

$39,99 €$

EBM 2020 Kommentar Innere Medizin mit allen Schwerpunkten

Kompakt: Mit Punktangaben, Eurobeträgen, Ausschlüssen, GOÄ

Hinweisen

Hermanns, Peter M. (Hrsg.)

XXII, 685 Seiten

$2020 \mid$ 1. Aufl. 2020

Springer-Verlag

978-3-662-61503-4 (ISBN)

$49,99 €$ 